\title{
How much insulin is enough? A quantitative assessment of the transdifferentiaton potential of liver
}

\author{
S. Perl • B. Hirshberg • D. M. Harlan • J. F. Tisdale
}

Received: 25 August 2006 / Accepted: 10 October 2006/ Published online: 13 January 2007

(C) Springer-Verlag 2007

To the Editor: Adenoviral vector-mediated expression of the pancreatic and duodenal homeobox 1 gene $(P d x l$; current designation in mice insulin promotor factor-1, Ipfl) in mice has previously been reported to result in hepatic, glucose-regulated insulin secretion sufficient to ameliorate experimentally induced diabetes [1]. In studies designed to assess the clinical applicability of this approach, we developed a protocol employing non-human primates rendered insulin-deficient (and therefore diabetic) through selective intra-arterial streptozotocin (STZ) infusion into the pancreas [2]. We obtained and amplified the adenoviral vector in which the CMV promoter drives rat $P d x I$ expression (Ad-CMV-Pdxl referred to hereafter as Ad$P d x 1$ ), kindly provided by S. Ferber, Endocrine Institute, Sheba Medical Centre, Tel-Hashomer, Israel.

In order to document vector activity prior to proceeding to the large animal model, we tested Ad- $P d x 1$ lots for activity in 8- to 10-week-old C57B16 mice (Jackson Laboratories, Bar Harbor, ME, USA), which were housed and handled in accordance with the guidelines set forth by the Committee on the Care and Use of Laboratory Animals of the Institute of Laboratory Animal Resources in a protocol approved by the Animal Care and Use Committee of the National Institute of Diabetes, Digestive, and Kidney

S. Perl $(\square) \cdot$ J. F. Tisdale

Molecular and Clinical Hematology Branch,

National Institute of Diabetes and Digestive and Kidney Diseases, National Institutes of Health,

Bethesda, MD, USA

e-mail: perls@niddk.nih.gov

B. Hirshberg • D. M. Harlan

Islet and Autoimmunity Branch, National Institute of Diabetes and Digestive and Kidney Diseases, National Institutes of Health, Bethesda, MD, USA
Diseases. Mice were rendered diabetic with STZ (200 mg/ kg i.p.). We treated 40 mice with the Ad- $P d x l$ vector, but compared with control mice, observed no improvement in glycaemia. All the mice lost significant weight and subsequently had to be killed (Fig. 1a), further supporting the absence of an insulin anabolic effect.

To test whether our negative results could be explained by the vector's failure to promote IpfI expression, we employed quantitative real-time PCR (qPCR) to assay for both Ipf1 and insulin, and western blotting to assess IPF1 protein expression. These assays clearly indicated vectordriven IpfI expression in the liver of Ad-Pdxl-treated mice; RNA was detected at a low PCR cycle number, and western blotting revealed IPF1 protein levels comparable to that from a rat insulinoma cell line (Fig. 1b,c). In order to further quantitate insulin gene expression, we removed approximately $25 \%$ of a control mouse liver, divided it into four quarters, and extracted RNA; one quarter was used as a negative control, and to the other three we added the RNA extracted from either 100,10 or 1 mouse islets. While insulin RNA from one islet was easily detectable (Table 1), that from Ad-Pdxl-treated mouse livers was at (shown) or below the limit of detection using 40 PCR cycles, similar to the negative control liver (final amplification plot in black). These results demonstrate that the hepatic insulin message induced by Ad- $P d x 1$ is less than that of one islet's RNA mixed with RNA from one-sixteenth of a liver. As four more PCR cycles were required to detect insulin message from the Ad-Pdxl-treated liver (assuming the signal represents specific amplification) compared with the cycle number when one islet was mixed with one-sixteenth of a control mouse liver, the Ad-Pdxl-induced insulin message was $2^{4}$ or 16 -fold less than the insulin message resulting from one islet in one-sixteenth of a liver control. Thus, the overall liver insulin RNA content induced by Ad- $P d x l$ is less than that of 1 islet equivalent. 
Fig. 1 We analysed 8- to 10week-old C57B16 mice that had been rendered diabetic by treatment with STZ $(200 \mathrm{mg} / \mathrm{kg}$ i.p.) and then been injected with $10 \mathrm{e}^{10}$ to $10 \mathrm{e}^{11}$ plaque-forming units of Ad- $P d x 1$ or monitored for evidence of liver to pancreas transdifferentiation. a Weights of Ad-Pdxl-treated diabetic mice (black circles) compared with untreated diabetic mice (grey squares) over 10 days. b Western blot for IPF1 protein detection in liver of treated $(+)$ and control (-) mice. RIN, rat insulinoma cell line serves as positive control. c Comparison of insulin message generated in the liver by Ad- $P d x I$ and the message generated by islets mixed in the liver at the indicated number (for details, see text). Open circles, one islet; open squares, ten islets; closed circles, 100 islets; grey squares, treared; black squares, control. $\Delta R n$, baseline subtracted fluorescent reading normalised to the reference dye

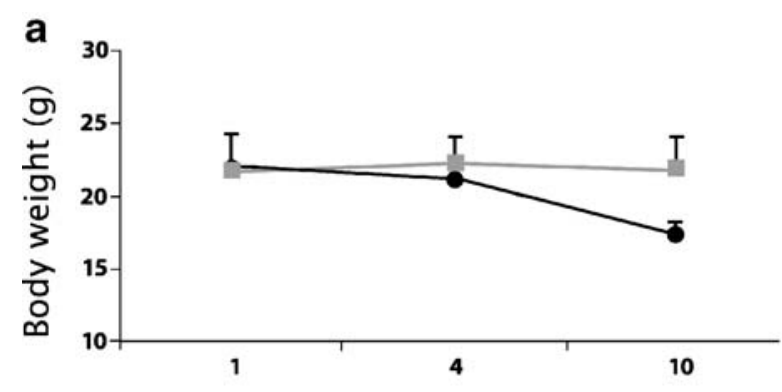

b

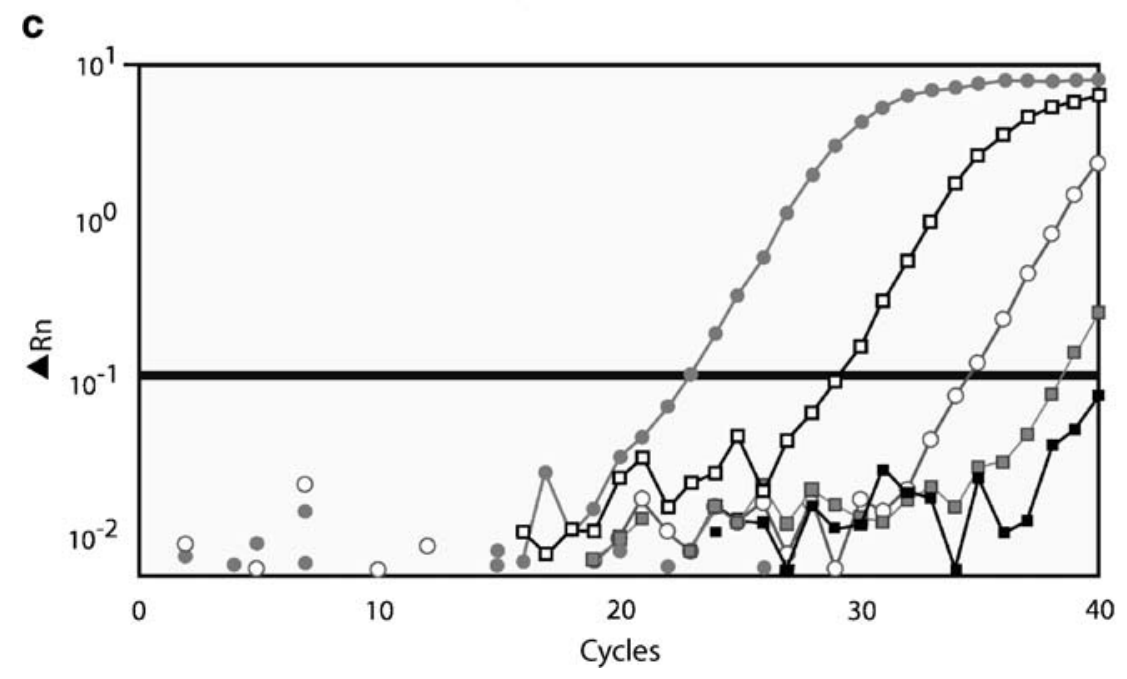

While Ipf1-induced hepatic insulin production (however minimal) is intriguing, these data are consistent with our in vivo results. That is, such negligible insulin production is unlikely to significantly improve glycaemia control. Ferber et al. also found only very low levels of hepatic insulin in their non-diabetic mice (far lower than that of the native pancreas) [1]. Together, these data suggest that an alternative explanation must be considered for the improved glycaemia reported in that study in diabetic animals treated with Ad-Pdxl. Indeed, some of our mice were also normoglycaemic, but only when fasted (they remained hyperglycaemic in the non-fasting state). We suggest that the hepatitis associated with Ad-Pdxl gene delivery [3] may have decreased mouse food intake over the brief period monitored. Certainly, before insulin treatment was available, starvation was the standard of care for diabetes, and

Table 1 Number of cycles required for detection of IpfI and $\beta$-actin gene expression in Ad- $P d x l$-treated and control mice using qPCR

\begin{tabular}{lll}
\hline mRNA & Untreated liver & $I P F-1$-treated liver \\
\hline$\beta$-Actin & $21-22$ & $21-22$ \\
$I p f 1$ & None & $22-24$ \\
\hline
\end{tabular}

while controlled starvation helped lower blood glucose and prolong life, the treatment was associated with inexorable wasting and death [4]. In their initial report [1], Ferber et al. did not record diabetic and control mouse weights, so we cannot address the possibility that weight loss explained their observations. In addition, the earlier report did not provide details on insulin expression in the diabetic mice. Interestingly, Kojima et al. recently reported liver insulin gene expression in mice with STZ-induced diabetes [5], raising the possibility that the liver retains cells capable of responding to hyperglycaemia, and this may have accounted for at least some of the effect reported by Ferber et al. We conclude that liver IpfI gene expression is insufficient to correct experimentally induced diabetes. Our results also demonstrate the vital importance of carefully quantifying gene expression [6] in pre-clinical as well as clinical studies related to potential gene therapy applications.

Acknowledgments This research was supported by the Intramural Research Program of the National Institute Diabetes and Digestive and Kidney disease, National Institutes of Health.

Duality of interest statement The authors state that they have no duality of interest. 


\section{References}

1. Ferber S, Halkin A, Cohen $\mathrm{H}$ et al (2000) Pancreatic and duodenal homeobox gene 1 induces expression of insulin genes in liver and ameliorates streptozotocin-induced hyperglycemia. Nat Med 6:568-572

2. Tal MG, Hirshberg B, Neeman $Z$ et al (2004) Induction of diabetes in nonhuman primates by means of temporary arterial embolization and selective arterial injection of streptozotocin. Radiology 230:163-168
3. Ber I, Shternhall K, Perl S et al (2003) Functional, persistent, and extended liver to pancreas transdifferentiation. J Biol Chem 278:31950-31957

4. Feudtner JC (2003) Bittersweet: diabetes, insulin, and the transformation of illness, vol 2. University of North Carolina Press, Chapel Hill

5. Kojima H, Fujimiya M, Matsumura K et al (2004) Extrapancreatic insulin-producing cells in multiple organs in diabetes. Proc Natl Acad Sci USA 101:2458-2463

6. Rajagopal J, Anderson WJ, Kume S et al (2003) Insulin staining of ES cell progeny from insulin uptake. Science 299:363 